\title{
Facebook y comunicación virtual. Un estudio experimental en docentes de educación superior
}

\section{Facebook and Virtual Communication. An Experimental Study in Higher Education Teachers}

\author{
Leydi Susan Fajardo Vizquerra \\ Universidad César Vallejo, Lima, Perú \\ ORCID: https://orcid.org/0000-0002-6141-7307 \\ Mitchell Alberto Alarcón Diaz*(i) \\ Universidad Nacional de Educación Enrique Guzmán y Valle, Lima, Perú \\ ORCID: https://orcid.org/0000-0003-0027-5701 \\ Noel Alcas Zapata \\ Universidad César Vallejo, Lima, Perú \\ ORCID: https://orcid.org/0000-0002-2505-6778 \\ Henry Hugo Alarcón Diaz \\ Universidad Nacional de Educación Enrique Guzmán y Valle, Lima, Perú \\ ORCID: https://orcid.org/0000-0003-1588-4390
}

Recibido 28-08-18 Revisado 09-09-18 Aprobado 12-12-18 En línea 17-12-18

*Correspondencia

Email: malarcond@ucv.edu.pe
Citar como:

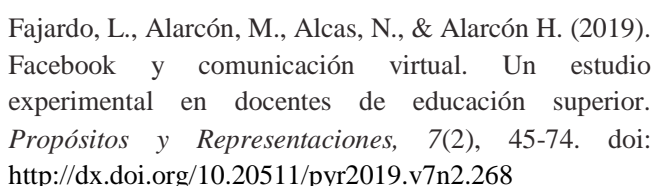

Fajardo, L., Alarcón, M., Alcas, N., \& Alarcón H. (2019). Facebook y comunicación virtual. Un estudio experimental en docentes de educación superior. Propósitos y Representaciones, 7(2), 45-74. doi: http://dx.doi.org/10.20511/pvr2019.v7n2.268 


\section{Resumen}

El presente estudio tuvo como objetivo determinar el efecto de un programa de capacitación Facebook en la comunicación virtual de docentes universitarios. La importancia de la temática desarrollada se fundamenta ya que en los momentos actuales, para el proceso de interacción y comunicación entre las comunidades laborales, académicas, económicas o sociales, se está haciendo uso de las Tecnologías de la Información y la Comunicación (TIC), en donde fluye todo tipo de información a través de las herramientas tecnológicas disponibles y redes sociales, y que es seleccionada por los usuarios, según el grupo de interés al que pertenecen; uno de ellos es Facebook. El enfoque fue cuantitativo, con diseño cuasi- experimental, para los cual se trabajó con una muestra de 140 docentes universitarios distribuida en dos grupos: control con 60 docentes y el segundo grupo experimental constituido por 80 docentes. Los resultados indican que existe influencia del programa de capacitación Facebook en la comunicación virtual de los docentes universitarios, como se demuestra con la prueba estadística ( $\mathrm{U}$ de Mann-Whitney $=155.500 ; \mathrm{Z}=$ -1.084; Sig. Asintótica (bilateral) .000<.05). Este hallazgo, puede ayudar a generar opiniones favorables para su posible utilización de la plataforma Facebook con fines de interacción social educativa.

Palabras clave: Facebook; Comunicación virtual; Educación superior; Interacción social.

\section{Summary}

The objective of this study was to determine the effect of a Facebook training program in the virtual communication of university teachers. The importance of the theme developed is based on the fact that at present, for the process of interaction and communication between labor, academic, economic or social communities, Information and Communication Technologies (ICT) are being used, where all types of information flow through the available technological tools and social networks, and which is selected by the users, according to the stakeholder to which they belong; one of them is Facebook. The approach was quantitative, with quasi-experimental design, for which a sample of 140 university teachers was divided into two groups: control group with 60 teachers and the experimental group with 80 teachers. The results indicate that there is influence of the Facebook training program in the virtual communication of university teachers, as demonstrated by the statistical test (Mann-Whitney $\mathrm{U}=155.500 ; \mathrm{Z}=-1,084$, Asymptotic (bilateral) significance $.000<.05$ ). This finding can help generate favorable opinions for its possible use of the Facebook platform for educational social interaction purposes.

Keywords: Facebook; Virtual Communication; Higher Education; Social Interaction.

\section{Introducción}

Los adelantos vertiginosos y trascendentes en la tecnología han afectado de manera significativa la sociedad actual. En este sentido, es imprescindible que la sociedad pueda administrar, comprender y evaluar adecuadamente la tecnología a la cual se tiene acceso. En esta línea de pensamiento, la educación en entornos virtuales particular, juega un rol muy importante, porque permite satisfacer las necesidades de aprendizaje de los estudiantes y por otro lado, mejora su rendimiento académico. Por lo tanto, las instituciones educativas del nivel superior universitario, deben proporcionar a sus estudiantes y docentes, el uso de tecnología para producir nuevos conocimientos, y estar acorde con los avances de las herramientas de la educación virtual. Desde 
esta perspectiva, la plataforma Facebook juega un rol importante para que los docentes universitarios, desarrollen en sus estudiantes el pensamiento crítico, posibiliten la resolución de problemas y la colaboración. Existen paralelamente, numerosas variables que de alguna manera, pueden afectar el proceso de formación de comunidades de aprendizaje, y entre los más comunes se tiene a la motivación y la satisfacción. Sobre la motivación Gutierrez (2016), sostuvo que, "la motivación es lo que las personas quieren hacer, decidir hacer, y cometer hacer" (p. 1). Es decir, previo a cualquier acción de la persona, habrá siempre cierta motivación intrínseca o extrínseca que lo impulsa a decidir hacer algo. En este sentido, la motivación es importante para el uso de cualquier herramienta virtual, y se convierte además de suma relevancia para el aprendizaje eficaz. Al respecto, Maslow (1991), sostuvo que "una teoría consistente de la motivación debería asumir, por el contrario, que la motivación es constante, inacabable, fluctuante y compleja, y que es una característica casi universal de prácticamente todos los estados organísmicos en cuestión" (p. 8). Lo cual implica que la complejidad que adquiere la motivación en las personas, depende de sus estados anímicos y hasta de los intereses y objetivos personales. En referencia a la satisfacción, Maslow (1991) afirmó que existen necesidades básicas y necesidades superiores. Las necesidades básicas constituyen el fundamento de un sistema intrínseco del ser humano, y considera entre ellas, las necesidades de seguridad, pertenencia, de amor y autoestima. Menciona también que las necesidades superiores se caracterizan porque deparan más felicidad y conducen a un crecimiento mayor de la persona, pero para que esto ocurra, se requiere de un entorno, más adecuado. El aprendizaje en línea y semipresencial, se han convertido en estos últimos años en una posibilidad para la enseñanza en el entorno de las comunidades de aprendizaje. En ese sentido, Kucuk y Sahin (2013), sostienen que el concepto de comunidades de aprendizaje, se ha fortalecido para aumentar su efectividad de estos entornos de aprendizaje.

Según el Observatorio de redes sociales, 2011, el auge de las mismas, durante los años 2009 y 2010, se catalogaron como una forma importante y efectiva para la comunicación entre sus usuarios. Entre las redes sociales encontramos especialmente al Facebook, que, de acuerdo a (Espuny, González, Lleixá \& Gisbert, 2011), en un estudio realizado con estudiantes universitarios, indican que es una red amigable y favorable entre los integrantes de una institución educativa para mantenerse permanentemente en comunicación, asimismo, amplía y diversifica los canales comunicativos. Ahora, es pertinente señalar que de acuerdo a Kolikant, 2010, la Web 3.0, se considera a la comunicación sostenida a través de las tecnologías y/o dispositivos móviles, lo que de una manera evita cualquier tipo de inconveniente. De esta manera, las barreras de espacio y tiempo se reducen, ya que estas nuevas formas de comunicarnos, han permitido cambios en nuestros roles rutinarios tanto en el nivel profesional y personal. "Si por algo se caracteriza la red en los últimos años es por la gran cantidad de herramientas que se han desarrollado y que permiten la participación y comunicación entre usuarios" (Gutiérrez, Román \& Sánchez, 2018, p.92).

Por otra parte, los docentes y estudiantes de una universidad, conforman en la práctica una red digital, que incluyen teléfonos móviles, internet, medios de comunicación. El Facebook previa capacitación, como una herramienta de aprendizaje de los docentes puede ayudar a que la comunicación digito virtual sea efectiva. Al respecto Davidovitch y Belichenko (2018), realizaron un estudio con estudiantes y se probó que los grupos de Facebook se utilizan para facilitar la comunicación, fomentar un clima social positivo, crear un diálogo y compartir material de aprendizaje entre los miembros del grupo. Asimismo, Hilscher (2013), sostuvo que las implicancias académicas del Facebook se evidencian en una comunidad virtual de aprendizaje, y los estudiantes universitarios sus docentes, la utilizan como una herramienta social en su entorno 
educativo. También, Mustafa y Emrah (2014), indicó que se probó a estudiantes de veinte países europeos, quienes se mostraron positivos o abiertos para la comunicación virtual y la posibilidad de la comunicación intercultural mediante los canales virtuales como el Facebook, MSN o Twitter. Otro estudio no menos relevante fue el de Shih (2013), afirmó que los estudiantes pueden mejorar su conocimiento profesional haciendo uso de otros medios complementarios a las clases como el Facebook, sino también en la evaluación de sus pares en este entorno virtual. Además, el uso de esta herramienta, puede mejorar el nivel de motivación y de aprendizaje de los estudiantes. En esta línea de pensamiento, el uso de la herramienta Facebook, se puede convertir en una estrategia de enseñanza - aprendizaje por parte del universitario, que podría generar mejores aprendizajes y en menor tiempo.

Al-Rawi (2018), realizó un estudio sobre comunidades en línea, haciendo uso de la plataforma Facebook. Los usuarios que participaron en esta experiencia fueron ciudadanos canadienses y posibles inmigrantes. Los resultados de este estudio indican que se evidencian sentimientos muy positivos hacia Canadá y sus ciudadanos y se indica además, que el administrador de Facebook opera como un guardián de acceso centralizado que filtra la comunicación en línea y direcciona la discusión hacia determinada dirección. Asimismo, Martin, Greiling y Wetzelhütter (2018), indicaron que un número creciente de empresas alemanas y austriacas, generan cuentas propias de Facebook para establecer comunicaciones con sus grupos de interés. Los hallazgos encontrados en 258 usuarios, indicaron los usuarios desean que la plataforma Facebook se convierta en un medio importante, para que les llegue, la mayoría de la información. También precisaron, que estos resultados permiten a los académicos y profesionales conocer con mayor profundidad, lo que ocurre en el Facebook, desde la perspectiva de los usuarios reales. Otro estudio cuasi experimental para indagar acerca de la efectividad de un método de enseñanza asistido por la plataforma Facebook, sobre la prevención del cáncer cervicouterino y dirigido a adolescentes de educación secundaria, fue realizado por Lai, et al (2015), quienes concluyeron que, la educación que se imparte de manera tradicional en la escuela puede ser efectiva, pero el método de discusión utilizando la plataforma Facebook, es más efectivo que la discusión en persona.

\section{Identidad social y redes sociales.}

La identidad parte del hecho de entender quiénes somos y cómo nos relacionamos con los demás, en este proceso, es necesario identificarnos con quienes nos comunicamos y asimismo, diferenciarnos respecto a ellos, lo cual evita confundirnos con los demás. A este hecho Íñiguez (2011) lo denomina como, dimensión experiencial de la identidad, también sostiene que "la singularidad, la unicidad, la exclusividad parecen ser características imprescindibles, al menos en nuestra cultura, de eso que llamamos identidad" (p. 3). Sin embargo, a estas características se le debe añadir en cierta continuidad en el tiempo, pero la temporalidad identitaria, se presenta nuevamente con el dilema igual o diferente, dado que el ser humano sabe que es la misma persona que fue en el pasado, pero al mismo tiempo se reconoce cambiada y diferente. También sostiene que, existe otro aspecto relevante de la identidad que no se refiere a la singularidad de la persona, sino más bien a la pluralidad del grupo o de la comunidad, y por complementariedad se habla de identidad social, la misma que en la práctica del lenguaje social contemporáneo se llama "redes".

En referencia a las redes sociales, tienen como una de sus mejores herramientas al Facebook, dado que crece en uso y funcionabilidad. Se incluye en este aspecto tres elementos claves para la interacción: un perfil construido en un sistema contenido, la capacidad de indicar o 
sugerir otros usuarios con quienes se puede establecer conexión y la capacidad de ver quienes están ya conectados con la persona que se desea establecer conexión (Boyd \&Ellison, 2007; Ahlqvist et al., 2000, citados por Lau, 2013, p. 18). Asimismo, asociados a estas tres capacidades, es necesario mencionar tres componentes esenciales para el éxito de las redes sociales: el contenido, la comunidad y web 2.0. En esta línea de pensamiento, la capacidad de crear comunidades es fundamental, para compartir contenidos con otras personas, usando la herramienta Facebook. Asimismo, las redes sociales en línea están siendo utilizadas por millones de personas, con el propósito de facilitar la comunicación y las conexiones con otros usuarios (Clark y Roberts, 2010, citados por Dickstein-Fischer, 2013, p. 9).

\section{Problemas que se originan en las redes sociales.}

Los problemas derivados en torno a las redes sociales se han evidenciado en los últimos años en la prensa, en donde son recurrentes: el acoso de todo tipo, la calumnia, la intimidación, que muchas veces genera inseguridad en los usuarios. Couros (2008) sostiene que, una vez que se elimina el temor a la seguridad, los sitios de redes sociales pueden abrir nuevos y amplios mundos de aprendizaje tanto para educadores como para estudiantes. Los docentes innovadores deben reconocer el potencial que contiene la herramienta Facebook para salvar las brechas culturales y al mismo tiempo, generar auténticos entornos de aprendizaje Tawney (2014), precisa que en las relaciones entre las personas en el Facebook, existen cuatro problemas que involucran a la persona humana, la relación, la comunicación y la romanticización.

\section{Participación estudiantil con la herramienta Facebook.}

La participación estudiantil ha evolucionado conjuntamente con la noción de como aprenden los estudiantes de educación superior universitaria. No se concibe, las disertaciones en las aulas con estudiantes pasivos, se ha integrado el desarrollo personal con el aprendizaje (Barber, 2012, citado por Sikes, 2015, p. 16). Es decir, se espera que los estudiantes participen plenamente sus experiencias universitarias para tener éxito académico, pero a la vez, que en un futuro inmediato tengan vidas exitosas, afectivas y comprometidas (ACPA, 1996, Keeling, 2004; 2006, citados por Sikes, 2015, p. 16).

Estudios realizados en referencia al uso de Facebook por parte de personas en edad universitaria, han revelado patrones de uso consistente y similar. Los estudiantes universitarios revisan sus cuentas de Facebook establecidas diariamente (Nadkarni \& Hofman, 2011; QuanHaase \& Young, 2011; Vitak et al., 2011, citados por Vigil \& Wu, 2015, p. 2) y permanecen entre 30 y 60 minutos por día en el sitio web (Ellison, Seinfield, \& Lempke, 2007; Ellison et al., 2011; Pempek et al., 2009; Quan-Haase \& Young, 2010, citados por Vigil \& Wu, 2015, p. 2). Uno de los sitios de redes sociales más populares y que tiene impacto en la educación es el Facebook, porque es una herramienta efectiva para impartir información y conocimiento, además pone mucho énfasis para la colaboración en línea y el intercambio de información debido a sus redes sociales, y sus características se centran en el usuario (Kalbande, et.al, 2012, citados por Parvez \& Akhtar, 2013, p. 2). Existen varias razones o motivos para que los usuarios participen en Facebook, una de ellas es la de mantener la comunicación entre ellos y sus familias; si los usuarios son estudiantes Facebook alienta la comunicación entre ellos y sus docentes, (Huang et al., 2010, citados por Aydin, 2012, p. 110). 
Por otra parte, en referencia a la evaluación de los estudiantes participantes, en el uso de la plataforma Facebook se puede abordar las necesidades de autoeficacia en estrategias de enseñanza únicas centradas en el estudiante. Los maestros y los miembros de la clase pueden usar el botón de superlativos o regalos virtuales para reconocer el logro de los alumnos en exhibiciones públicas, privadas o anónimas (Bowers-Campbell, 2008). En los estudiantes universitarios, es más interesante y sencillo implementar estrategias de enseñanza aprovechando su compromiso. Charlton, Devlin \& Drummond (2009), sostienen que el Facebook reemplaza con eficacia a otras tecnologías de las redes sociales. Con base en estos hallazgos, hemos desarrollado una herramienta llamada CommonGround, diseñada para ejecutarse en la plataforma de Facebook, que aprovecha el compromiso de los estudiantes con el servicio y uniendo las características de comunicación y 'conciencia social' inherentes a dicha plataforma. Charnigo \& Barnett-Ellis (2007) precisaron que la plataforma Facebook, proporciona un nivel sofisticado de conectividad lo cual es un beneficio para los usuarios, y mantiene también un seguimiento diario de los intereses populares (por ejemplo, libros favoritos) y datos demográficos de los miembros (número de miembros, orientación política) y los compara con promedios generales de miembros de Facebook. DePe (2011), al referirse a los espacios disponibles en las redes sociales, y en particular en el Facebook, indica que pueden participar estudiantes universitarios que pertenecen a culturas periféricas especialmente a aquellos que pueden estar físicamente aislados de otros y que comparten esa cultura. La Internet con su herramienta Facebook, puede ser un medio principal para estas personas para conectarse con aquellos a quien identifican. Asimismo, es un foro para que se posicionen en el mundo haciendo argumentos sobre cómo quieren que otros los perciban.

\section{Comunicación sincrónica.}

El aprendizaje en línea es una posibilidad que tienen actualmente los estudiantes universitarios, para su aprendizaje. Sin embargo, existen algunas observaciones, en el sentido que este tipo de comunicación no ayuda mucho para la reflexión y el pensamiento crítico. Pero es una muy buena posibilidad, para el acceso que debe tener los estudiantes universitarios sobre áreas específicas de sus programas de su formación académica. En ese sentido, la comunicación sincrónica es entendida como la interacción entre dos o más personas en forma simultánea y que se puede concretar en físico o en virtual. En referencia a la comunicación virtual con esta característica se utilizan algunas herramientas disponibles en la actualidad: videos, audio entre otros. En ese sentido, Breffni O’Rourke, \& Stickler, (2017) definen la comunicación sincrónica como comunicación dialógica que se concreta a partir de la presencia simultánea (copresencia) en un especio comunicativo compartido. También, Huang (2018) afirma que el aprendizaje colaborativo asistido por computadora facilita por ejemplo, la extensión de la adquisición de un segundo idioma, porque la comunicación se da en tiempo real, mediante la comunicación de texto, audio y vídeo. Asimismo, Ko (2015), sostiene que desde una perspectiva teórica, la colaboración respaldada por computadora sobre la base del software grupal, proporciona a los individuos la posibilidad de un mayor nivel de intercambio de información coordinación y navegación, mejorando la participación equitativa. Asimismo, Rochester (2017) plantea que lo asincrónico asume la colaboración, permite el desarrollo del pensamiento crítico, y por consiguiente acceder a niveles altos de aprendizaje y conocimiento, estimulando la memoria de largo plazo.

\section{Comunicación asincrónica.}

La comunicación asincrónica, por otra parte, implica que los estudiantes interactúen en momentos y formas diferentes (foros, correos, etc.). Al respecto, Giesbers, Rienties, Tempelaar \& Gijselaers 
(2013), sostienen que lo sincrónico y asincrónico favorece el compromiso y el aprendizaje del estudiante.

También, Maushak \& Ou (2007), sostienen que la comunicación en comunidades asincrónicas tiene ventajas sobre la comunicación en comunidades sincrónicas fundamentalmente en la educación a distancia. Es decir en la comunicación asincrónica se proporciona a los estudiantes, además del conocimiento la posibilidad de tomarse más tiempo para la reflexión crítica. Asimismo, Wu (2018), sostuvo que con la comunicación asincrónica ayudan a alinear las posturas de cada estudiante. El aprendizaje en línea, es una necesidad que tienen las universidades para lograr que algunas veces los estudiantes no abandones sus estudio presenciales. Por esta razón, Kebble (2017), sostuvo que el ambiente de aprendizaje en línea, fomenta un sentido de conexión y asimilación que puede mejorar la experiencia de aprendizaje y compromiso de una persona.

\section{Método}

La investigación se diseñó con el propósito de determinar el efecto de un programa de capacitación Facebook en la comunicación virtual de los docentes de una universidad privada de Lima (Perú) haciendo uso de la plataforma Facebook. Para la elaboración del programa se siguieron las siguientes fases: Planificación, en donde se organizó la convocatoria, presentación del taller de capacitación y beneficios del mismo. Asimismo, se le proporcionó los materiales para la realización del taller de capacitación y se dispuso un lugar donde se llevaría a cabo. En cuanto a la ejecución: se desarrolló el taller de capacitación virtual en cuatro días, cuatro sesiones dentro de los ambientes de la universidad privada. Finalmente, en la fase de evaluación, se utilizó la técnica de la observación, mediante una lista de cotejo.

La capacitación Facebook, brindó información sobre los diferentes servicios web 2.0 que existen en el medio y que favorecen la interacción docente-alumno, ahondando en las principales características de uno centrado en la comunicación virtual. Asimismo, teniendo como objetivo específico capacitar a los docentes participantes en el manejo de herramientas web 2.0 para su aplicación en el desarrollo o gestión de sus asignaturas, incidiendo en el uso de Facebook y por otra parte, que conozcan los diferentes tipos de servicios que brinda la web 2.0 en el campo de la enseñanza - aprendizaje y manejar las opciones principales de privacidad y comunicación de Facebook.

La capacitación Facebook fue de modalidad práctica, por lo cual las actividades se desarrollaron con carácter aplicativo. Se utilizaron métodos didácticos tales como: dinámicas grupales, trabajos individuales, participación activa en las sesiones y fuera de ellas. Los medios y materiales utilizados fueron: presentaciones, videos, retroproyector, recursos web, lecturas, entre otros.

El diseño fue cuasi experimental, con un grupo de control de 60 docentes y otro grupo experimental de 80 docentes, el cual fue capacitado. La medición de la variable comunicación virtual se realizó mediante dos dimensiones: Comunicación sincrónica con el indicador: Comunicación en tiempo real Mensajes/chat y la dimensión Comunicación asincrónica con tres indicadores: Comunicación en tiempos distintos, Tareas con plazos, Mensajes. Se utilizó la técnica de la observación, mediante una lista de cotejo con 18 ítems. 


\section{Resultados}

Los niveles de participación de los docentes, fue muy significativo, y se realizó en horarios que se ajustaron a su disponibilidad. Con relación a la variable comunicación virtual, los dos grupos (de control y experimental) se les aplicó un pretest antes del inicio del programa de capacitación Facebook y el postest al finalizar dicho programa. Asimismo, se realizó la evaluación teniendo en cuenta las dimensiones: comunicación sincrónica y comunicación asincrónica, que se detallan a continuación:

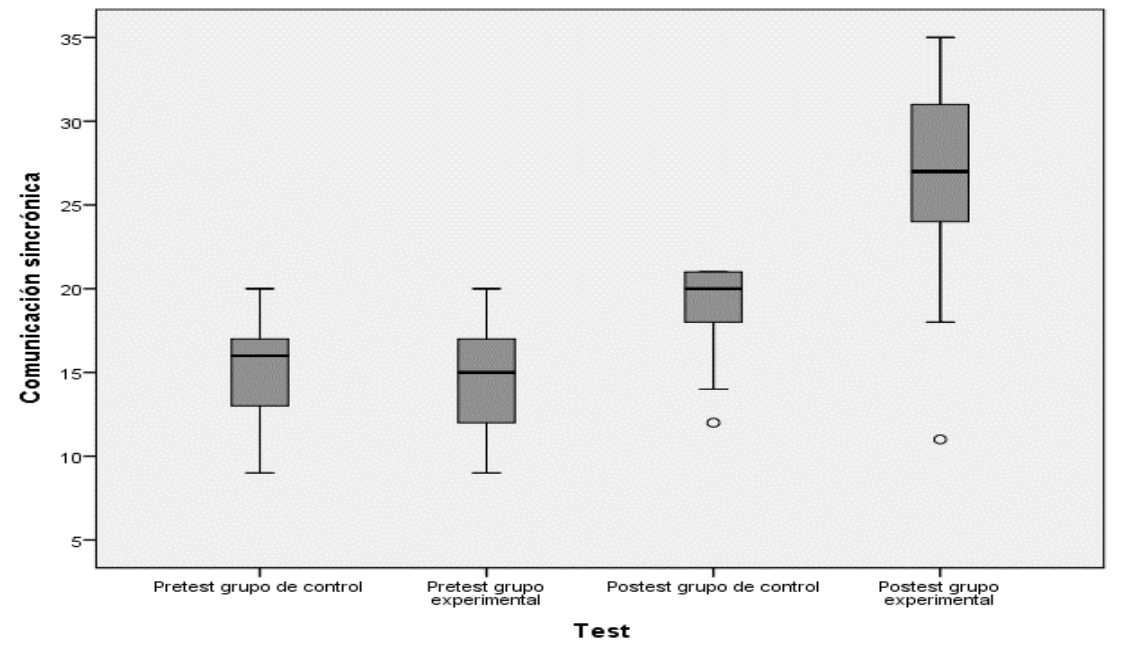

Figura 1. Diagrama de cajas entre los grupos: control y experimental para la comunicación sincrónica.

Como se observa en la figura 1, al aplicarse el pretest a ambos grupos, se evidencia que la mediana del grupo de control es igual a 16, en cambio la mediana del grupo experimental equivale a 15. Asimismo, en referencia a la prueba de postest, se observa que el grupo de control presenta una mediana igual a 20, mientras que el grupo experimental tiene una mediana igual a 27.

Tabla 1.

Estadístico de prueba para el pretest de la dimensión comunicación sincrónica.

Estadísticos de prueba $^{\mathrm{a}} \quad$ Comunicación sincrónica

U de Mann-Whitney

2144.000

W de Wilcoxon

5384.000

$\mathrm{Z}$

Sig. asintótica bilateral)

.278

a. Variable de agrupación: Test 
En la tabla 1, se observa que el estadístico de prueba U de Mann-Whitney, indica que no existen diferencias significativas en los resultados del pretest entre los grupos de control y experimental en relación a la dimensión comunicación sincrónica (U de Mann-Whitney = 2144.000; Z $=-1.084$; Sig. asintótica (bilateral) $=0.278>.05$ ). Este resultado corrobora lo que gráficamente expresa la figura 1.

Tabla 2.

Estadístico de prueba para el postest de la dimensión comunicación sincrónica.

\begin{tabular}{ll}
\hline Estadísticos de prueba $^{\mathrm{a}}$ & Comunicación sincrónica
\end{tabular}

$\begin{array}{ll}\text { U de Mann-Whitney } & 263.500\end{array}$

$\begin{array}{ll}\text { W de Wilcoxon } & 2093.500\end{array}$

Z -9.033

Sig. Asintótica (bilateral) $\quad .000$

a. Variable de agrupación: Test

En la tabla 2, se observa que el estadístico de prueba U de Mann-Whitney, indica que existen diferencias significativas en los resultados del postest entre los grupos de control y experimental en relación a la dimensión comunicación sincrónica ( $U$ de Mann-Whitney = 263.500; $Z=-9.033 ;$ Sig. asintótica (bilateral) $=0.000<.05$ ). Este resultado corrobora lo que gráficamente expresa la figura 1.

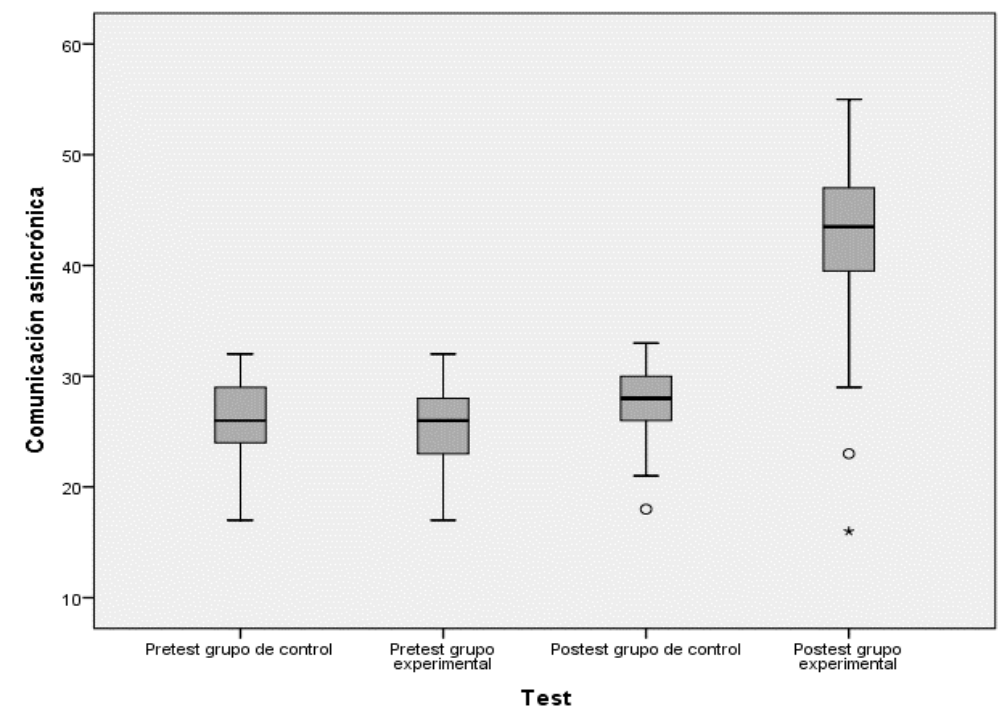

Figura 2. Diagrama de cajas entre los grupos: control y experimental para la comunicación asincrónica.

Como se observa en la figura 2, al aplicarse el pretest, se evidencia que la mediana para ambos grupos (control y experimental) es igual a 25 , en cambio en la prueba del postest, se 
observa que el grupo de control presenta una mediana igual a 28 , mientras que el grupo experimental tiene una mediana igual a 44.

Tabla 3.

Estadístico de prueba para el pretest de la dimensión comunicación asincrónica.

\begin{tabular}{lc}
\hline Estadísticos de prueba $^{\mathrm{a}}$ & Comunicación asincrónica \\
\hline U de Mann-Whitney & 2166.000 \\
W de Wilcoxon & 5406.000 \\
$\mathrm{Z}$ & -.990 \\
Sig. asintótica(bilateral) & .322 \\
\hline a. Variable de agrupación: Test &
\end{tabular}

a. Variable de agrupación: Test

En la tabla 3, se observa que el estadístico de prueba U de Mann-Whitney, indica que no existen diferencias significativas en los resultados del pretest entre los grupos de control y experimental en relación a la dimensión comunicación asincrónica ( $\mathrm{U}$ de Mann-Whitney = 2166.000; $Z=-.990 ;$ Sig. asintótica (bilateral) $=0.322>.05$ ). Este resultado corrobora lo que gráficamente expresa la figura 2 .

Tabla 4.

Estadístico de prueba para el postest de la dimensión comunicación asincrónica.

Estadísticos de prueba $^{\mathrm{a}} \quad$ Comunicación asincrónica

$\begin{array}{lc}\text { U de Mann-Whitney } & 161.000 \\ \text { W de Wilcoxon } & 1991.000 \\ \text { Z } & -9.438 \\ \text { Sig. asintótica (bilateral) } & .000\end{array}$

a. Variable de agrupación: Test

En la tabla 4, se observa que el estadístico de prueba U de Mann-Whitney, indica que existen diferencias significativas en los resultados del postest entre los grupos de control y experimental en relación a la dimensión comunicación asincrónica ( $\mathrm{U}$ de Mann-Whitney = 161,$000 ; Z=-9.438$; Sig. asintótica (bilateral) $=.000<.05$ ). Este resultado corrobora lo que gráficamente expresa la figura 2 . 


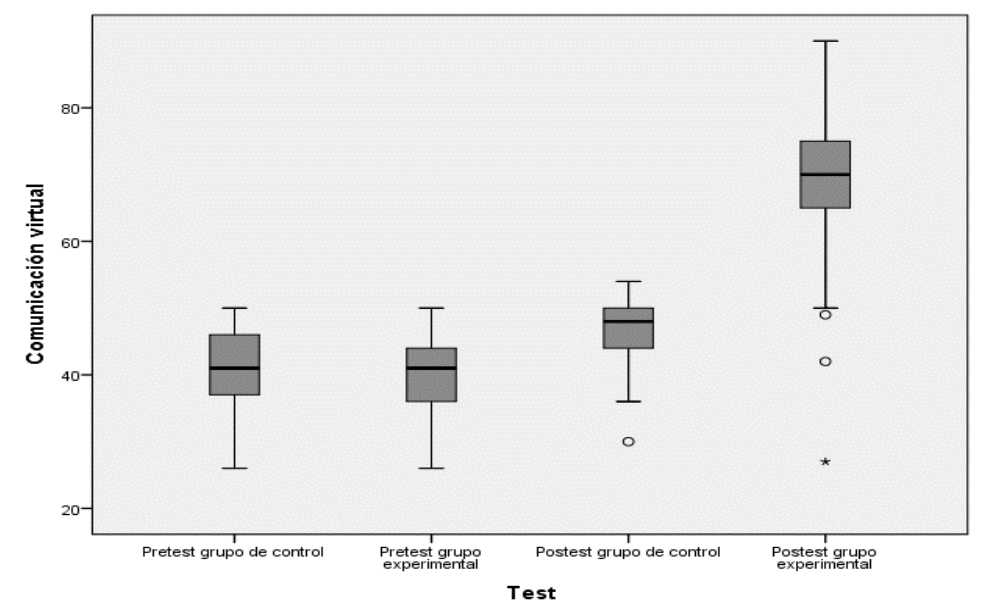

Figura 3. Diagrama de cajas entre los grupos: control y experimental para la comunicación virtual.

Como se observa en la figura 3, al aplicarse el pretest, se evidencia que la mediana de la variable comunicación virtual para ambos grupos (control y experimental) es igual a 40 en cambio en la prueba del postest, se observa que el grupo de control presenta una mediana igual a 44, mientras que el grupo experimental obtiene una mediana igual a 70 .

\section{Tabla 5.}

Estadístico de prueba para el pretest de la variable comunicación virtual.

\begin{tabular}{lc}
\hline Estadísticos de prueba $^{\mathrm{a}}$ & Comunicación virtual \\
\hline U de Mann-Whitney & 2134.000 \\
$\mathrm{~W}$ de Wilcoxon & 5374.000 \\
$\mathrm{Z}$ & -1.125 \\
Sig. asintótica (bilateral) & .261
\end{tabular}

a. Variable de agrupación: Test

En la tabla 5, se observa que el estadístico de prueba U de Mann-Whitney, indica que no existen diferencias significativas en los resultados del pretest entre los grupos de control y experimental en relación a la variable comunicación virtual (U de Mann-Whitney $=2134.000$; Z $=-1.125$; Sig. asintótica $($ bilateral $)=0.261>.05$ ). Este resultado corrobora lo que gráficamente expresa la figura 3. 


\section{Tabla 6.}

Estadístico de prueba para el postest de la variable comunicación virtual

\begin{tabular}{lc}
\hline Estadísticos de prueba $^{\mathrm{a}}$ & Comunicación virtual \\
\hline U de Mann-Whitney & 155.500 \\
W de Wilcoxon & 1985.500 \\
$\mathrm{Z}$ & -9.458 \\
Sig. sintótica(bilateral) & .000
\end{tabular}

a. Variable de agrupación: Test

En la tabla 6, se observa que el estadístico de prueba U de Mann-Whitney, indica que existen diferencias significativas en los resultados del postest entre los grupos de control y experimental en relación a la variable comunicación virtual (U de Mann-Whitney = 155,500; C; Sig. asintótica (bilateral) $=.000<.05)$. Este hallazgo, corrobora lo que gráficamente expresa la figura 3.

\section{Discusión}

El hecho de que la media aritmética y el análisis inferencial resultaran a favor del grupo experimental después de la intervención, nos permite asumir que el empleo metódico y sistemático del facebook mejora la comunicación virtual de los docentes universitarios (U de Mann-Whitney $=155,500 ; C ;$ Sig. asintótica (bilateral) $=.000<.05)$. También a través del análisis estadístico realizado, se corroboró la influencia del facebook en las dimensiones sincrónica (U de Mann-Whitney $=263,500 ; Z=-9.033 ;$ Sig. asintótica (bilateral) $=.000<.05)$ y asincrónica $(U$ de Mann-Whitney $=161.000 ; Z=-9.438 ;$ Sig. asintótica (bilateral) $=.000<.05$ ).

Estos resultados coinciden con las premisas sostenidas por Ellis (2007), Davidovitch y Belichenko (2018), Espuny, González, Lleixá y Gisbert (2011), Kucuk y Sahin (2013) quienes afirmaron que la conectividad, comunicación y efectividad se consolidan como un aporte valioso ofrecido por la red social creada por Mark Zuckerberg. En ese sentido, la propuesta de la aplicación institucional en favor de la mejora de la comunicación entre los miembros de la comunidad universitaria a través del facebook, se presenta como una oportunidad para vincular los intereses académicos de los docentes, así como el compromiso y el aprendizaje de los estudiantes (Huang et al., 2010, citados por Aydin, 2012 y Giesbers, Rienties, Tempelaar y Gijselaers (2013). Esto a decir de Ko (2015), refuerza la postura teórica la cual valora el impacto que tienen las nuevas tecnologías al incrementar las posibilidades de participación colectiva de los usuarios.

\section{Referencias}

Al-Rawi, A. (2018). Facebook and virtual nationhood: social media and the Arab Canadians community. AI \& Society, (Preprints), 1-13. Doi: https://doi.org/10.1007/s00146-0170742-3 
Aydin, S. (2012). A review of research on facebook as an educational environment. Educational Technology, Research and Development, 60(6), 1093-1106. Doi: http://dx.doi.org/10.1007/s11423-012-9260-7

Breffni O'Rourke, \& Stickler, U. (2017). Synchronous communication technologies for language learning: Promise and challenges in research and pedagogy. Language Learning in Higher Education, 7(1), 1-20. Doi: http://dx.doi.org/10.1515/cercles-2017-0009

Bowers-Campbell, J. (2008). Cyber "pokes": Motivational antidote for developmental college readers. Journal of College Reading and Learning, 39(1), 74-87. Doi: https://doi.org/10.1080/10790195.2008.10850313

Charlton, T., Devlin, M., \& Drummond, S. (2009). Using "Facebook" to improve communication in undergraduate software development teams. Computer Science Education, 19(4), 273-292. Doi: https://doi.org/10.1080/08993400903384935

Charnigo, L., \& Barnett-Ellis, P. (2007). Checking out Facebook.com: The impact of a digital trend on academic libraries. Information Technology and Libraries, 26, 23-34. Doi: https://doi.org/10.6017/ital.v26i1.3286

Couros, A. (2008). Safety and social networking: How can we maximize the learning power of participatory web sites while ensuring students are protected and behave responsibly? Technology and Learning, 28(7), 20. Recuperado de: https://eric.ed.gov/?id=EJ788845

Davidovitch, N., \& Belichenko, M. (2018). Facebook Tools and Digital Learning Achievements in Higher Education. Journal of Education And E-Learning Research, 5(1), 8-14. Doi: https://doi.org/10.20448/journal.509.2018.51.8.14

DePew, K. E. (2011). Social media at academia's periphery: Studying multilingual developmental writers' Facebook composing strategies. Reading Matrix: An International Online Journal, 11(1), 54-75. Recuperado de: https://digitalcommons.odu.edu/english_fac_pubs/29/

Dickstein-Fischer, L. (2013). Facebook use in relation to gender, introversion- extroversion, and sense of belonging among college students (Order No. 3524489). Recuperado de: https://repository.library.northeastern.edu/files/neu:969/fulltext.pdf

Espuny, C., González, J., Lleixá, M., \& Gisbert, M. (2011). Actitudes y expectativas del uso educativo de las redes sociales en los alumnos universitarios. Revista de Universidad y Sociedad del Conocimiento, 8(1), 171-185. Doi: https://doi.org/10.7238/rusc.v8i1.839

Giesbers, B., Rienties, B., Tempelaar, D., \& Gijselaers, W. (2013). Investigating the relations between motivation, tool use, participation, and performance in an e- learning course using web - videoconferencing. Computers in Human Behavior, 29(1), 285-292. Doi: https://doi.org/10.1016/j.chb.2012.09.005

Gutiérrez, I., Román, M., \& Sánchez, M. (2018). Estrategias para la comunicación y el trabajo colaborativo en red de los estudiantes universitarios. Comunicar, 26(54), 91-100. Doi: https://doi.org/10.3916/C54-2018-09

Gutierrez, K. (2016). Designing for motivation: Three theories eLearning designers can use. Technology Enabled Learning Excellence Essentials. Recuperado de: https://www.hr.com/en/topleaders/all_articles/designing-for-motivation-three-theorieselearning-_iqbzlikm.html

Hilscher, J. (2013, January 1). A Case Study Examining How Students Make Meaning out of Using Facebook as a Virtual Learning Community at a Midwestern University. ProQuest $L L C$. Recuperado de: https://lib.dr.iastate.edu/cgi/viewcontent.cgi? article $=4041 \&$ context $=$ etd

Huang, X. (2018). Improving Communicative Competence through Synchronous Communication in Computer-Supported Collaborative Learning Environments: A 
Systematic Review. Education Sciences, 8(1), 15. Doi: https://doi.org/10.3390/educsci8010015

Íñiguez, L. (2001). Identidad: De lo Personal a lo Social. Un Recorrido Conceptual. En Eduardo Crespo (Ed.), La constitución social de la subjetividad. (p. 209-225). Madrid: Catarata.

Kebble, P. G. (2017). Assessing Online Asynchronous Communication Strategies Designed to Enhance Large Student Cohort Engagement and Foster a Community of Learning. Journal of Education and Training Studies, 5(8), 92-10. Doi: https://doi.org/10.11114/jets.v5i8.2539

Ko, C. (2015). The Provision of Feedback Types to EFL Learners in Synchronous Voice Computer Mediated Communication. Research-Publishing.Net.

Kolikant, D. (2010). Digital natives, better learners? Students' beliefs about how the Internet influenced their ability to learn. Computers in Human Behavior, 26(6), 1384-1391. Doi: https://doi.org/10.1016/j.chb.2010.04.012

Kucuk, S., \& Sahin, I. (2013). From the perspective of community of inquiry framework: An examination of facebook uses by pre-service teachers as a learning environment. TOJET: The Turkish Online Journal of Educational Technology, 12(2) Recuperado de: https://eric.ed.gov/?id=EJ1015425

Lai, C., Wu, W., Tsai, S., Cheng, S., Lin, K., \& Liang, S. (2015). The Effectiveness of a FacebookAssisted Teaching Method on Knowledge and Attitudes about Cervical Cancer Prevention and HPV Vaccination Intention among Female Adolescent Students in Taiwan. Health Education \& Behavior, 42(3), 352-360. Doi: https://doi.org/10.1177/1090198114558591

Lau, A. R. (2013). Facebook as a facilitator of organizational identification in colleges and universities: Exploring relationships among educational institutions, student tenure, and interaction with multiple organizational targets. Recuperado de: https://dc.uwm.edu/cgi/viewcontent.cgi?referer=https://scholar.google.com.pe/\&httpsre dir $=1 \&$ article $=1131 \&$ context $=$ etd

Martin, S., Greiling, D., \& Wetzelhütter, D. (2018). Expectations of Facebook users towards a virtual dialogue with their public utility. International Journal of Energy Sector Management, 12(3), 408-425. Doi: https://doi.org/10.1108/IJESM-11-2017-0013

Maslow, A. H. (1991). Motivación y personalidad. Madrid, España: Ediciones Díaz de Santos, S. A.

Maushak, N. J., \& Ou, C. (2007). Using synchronous communication to facilitate graduate students' online collaboration. Quarterly Review of Distance Education, 8(2), 161-169.

Mustafa, Y., \& Emrah, A. (2014). An Intercultural Communication Training Test in a Special Case of Europe (An Applied Field Study Through the Instrument of Virtual Conduit). Procedia Social And Behavioral Sciences, 141(1), 1068-1073.Doi: https://doi.org/10.1016/j.sbspro.2014.05.179

Observatorio de Redes Sociales (2011). Informe de resultados del Observatorio de Redes Sociales, 3 a oleada. The Cocktail Analysis.

Parvez, A., \& Akhtar Hussain, M. A. (2013). Use of web 2.0 in saudi arabia universities. International Journal of Information Dissemination and Technology, 3(3), 158-166. Recuperado de: http://www.indianjournals.com/ijor.aspx?target=ijor:ijidt $\&$ volume=3\&issue=3\&article= 003

Rochester, M. R. (2017, January 1). Synchronous Communication and Its Effects on the Collaboration of Professional Workplace Employees Engaged in a Problem Activity. ProQuest LLC, 
Shih, R. (2013). Effect of Using Facebook to Assist English for Business Communication Course Instruction. Turkish Online Journal of Educational Technology - TOJET, 12(1), 52-59. Recuperado de: https://www.learntechlib.org/p/132122/

Sikes, S. M. (2015). Facebook is my extracurricular life!: A phenomenological examination of undergraduate facebook usage and student involvement. Estados Unidos: ProQuest Central.

Tawney, J. J. (2014, Jul). The fourfold problems of Facebook. New Oxford. Recuperado de: https://www.newoxfordreview.org/documents/the-fourfold-problems-of-facebook/

Vigil, T. R., \& Wu, H. D. (2015). Facebook users' engagement and perceived life satisfaction. Media and Communication, 3(1), 05-16. Doi: http://dx.doi.org/10.17645/mac.v3i1.199

Wu, Z. (2018). Positioning (Mis) Aligned:The (Un)Making of Intercultural Asynchronous Computer-Mediated Communication. Language Learning \& Technology, 22(2), 75-94. Recuperado de: https://eric.ed.gov/?id=EJ1181217 